\section{Kaleidoscope}

Derek K. Tracy, Dan W. Joyce,

Sukhwinder S. Shergill

June 27 this year was the day that funding was approved for San Francisco's Golden Gate Bridge physical suicide deterrent barrier (www.ggbsuicidebarrier.org). This follows over 1400 suicides since the bridge's opening in 1937: a morbid record 46 were in 2013 alone, possibly exacerbated by the global economic crisis. The barrier is in place to prevent the end-point of suicidal behaviour (physically preventing suicide) but how much effort is going into the examination of the external factors that drive such behaviour? Aleman \& Denys ${ }^{1}$ argue that psychiatry has failed to tackle suicide as a disease entity in its own right, instead relegating the act of deliberately ending one's life to a symptom or consequence of an underlying psychiatric illness. They note that in DSM-5 suicidality is only mentioned as a symptom of borderline personality disorder and mood disorders, despite this presenting as the most prominent psychiatric emergency. They argue for a more experimental approach based on the National Institute of Mental Health's Research Domain Criteria (www.nimh.nih.gov/research-priorities/ rdoc/index.shtml) that emphasises the study of psychiatric phenomena with reference to their underlying mechanisms: in this case, processing of negative valence, context and response selection, and mechanisms to regulate arousal.

Significant adverse childhood experiences have been estimated to have a role in up to a third of mental illnesses worldwide. Few adults have escaped some form of childhood bullying, and it forms a common thread in the personal narratives of many of our patients' developmental histories; but how does it contribute to later mental ill-health? Considerably, according to a large 50 -year prospective British cohort study ${ }^{2}$ that tracked mental health outcomes in almost 8000 individuals. Exposure to frequent bullying between the ages of 7 and 11 years resulted in greater psychological difficulties up to 40 years after these events. This was characterised by increased psychological distress with higher rates of depression, anxiety and suicidality; a detrimental impact on social function indexed by fewer relationships and increased economic hardship; and a worse perceived quality of life. The authors conclude that significant bullying is analogous to children being placed in care, and these data demand that active interventions are implemented to reduce bullying exposure in children within these age groups.

What is the mechanism that links such adversity with cognitive change and subsequent action? Neuroimaging data ${ }^{3}$ show that childhood maltreatment is significantly associated with later-life reductions in grey matter volumes in the limbic structures of the hippocampus, parahippocampus and anterior fusiform gyrus. Later-life brain changes following childhood maltreatment have been previously demonstrated but typically have been confounded by the comorbidity of substance misuse, which is twice as common than in those without such neglect. The specific hippocampal regions showing changes are critically involved in neurogenesis and regulation of the hypothalamic pituitary axis, and have previously been linked with the development of an anxious temperament. Intriguingly, although childhood maltreatment increases the risk of later-life substance misuse, this work indicates parallel processes such that later neuropathological damage is an independent predictor of regional anatomical change - and comorbidity leads to even greater additive neuronal losses. The medial temporal cortical regions identified in these studies have also been identified in studies of behavioural change following neurosurgery; tasks requiring interpretation of emotions and the intentions of others at a basic level were more affected by temporo/insular lesions, with emotion recognition being maximally impaired by anterior temporal and amygdalar lesions. ${ }^{4}$ In contrast, tasks relying on higher-level mentalisation, including consideration of others' perspectives, were maximally affected by prefrontal lesions. This hierarchical approach provides a useful heuristic in understanding the complex mechanisms underlying social behaviour.

Along the same theme of interacting reward processing and underlying brain systems, the use of pornography has been shown to be associated with regional cortical change. This is an important issue given the increasing access to pornography offered by the internet and against a background of lively debate about the consequences of such objectification of (predominantly) women, contrasted by libertarian views about press freedom and the freedom to choose what to do with one's body. However, to date, there have been little hard data describing its effects on the brain, although there is one study in macaque monkeys demonstrating that male monkeys were willing to give up juice rewards to watch pictures of female monkeys' genitalia. Filling this gap, a German neuroimaging study ${ }^{5}$ has shown a significant negative association between the number of hours of pornography consumed by participants and grey matter volume in the striatum, with reduced functional connectivity between this reward-processing region and the higher-order dorsolateral prefrontal cortex. While the direction of causality is not clear (does watching pornography damage the brain's reward system, or do some brain types prefer to watch?), longitudinal studies present unique recruitment, logistical and ethical challenges.

Deciding on the best location to convene a Kaleidoscope editorial meeting is not an easy call. There's a tension between the joy in repetition - a return to previous venues with the benefit of known, reliable seating and a consistent coffee experience $-v$. the potential of discovering newer venues with better baristas, deliberately design-conscious uncomfortable furniture and the alluring promise of obscure boutique coffee blends. What makes one prefer to exploit existing strategies over exploring newer perhaps better - options? In the past two decades, the Bayesian model of human inferential reasoning has dominated cognitive science: in this model, evidence for hypotheses or actions (go to coffee shop A or B) are assigned 'weights' expressed as prior probabilities (previous experience informs that coffee shop A has more comfortable seating than B), and these are combined with the likelihood model, which summarises new incoming information or evidence (the new coffee at B tastes fantastic). Reasoning proceeds by multiplying the prior probabilities by the likelihood, effectively representing our updating of knowledge of the world (boutique coffee shops) and informing our decision (visit the coffee shop with this highest posterior probability).

The problem is that even with trivial numbers of candidate strategies/actions and contexts (collectively called the dimensionality of the problem) a Bayesian formulation leads to exponential growth in computational demand - often due to additional arithmetic to ensure that the laws of probability are obeyed - which the applied mathematician Richard Bellman called 'the curse of dimensionality'. Donoso, Collins and Koechlin ${ }^{6}$ propose a solution in a recent paper in Science: their model of hypothesis testing and strategy 
selection in the human prefrontal cortex allows for a number of strategies to be held online (in a 'buffer') and evaluated for reliability - how well they perform in terms of action outcomes and how well it matches the context (or cognitive state) of the required decision. Their corresponding neuroimaging data show that the basal ganglia/ventral striatum is active in evaluating performance, anterior ventromedial prefrontal areas activate in evaluating the reliability of the available strategies while posterior prefrontal area predicted when strategies should be switched for exploration.

In the end, the curse of dimensionality rendered our editorial meeting moot - estimating it would take eight calendar months to calculate the required probabilities - favouring the wellestablished strategy of an email exchange with each member brewing their own coffee.
1 Aleman A, Denys D. A road map for suicide research and prevention. Nature 2014; 509: 421-3.

2 Takizawa R, Maughan B, Arseneault L. Adult health outcomes of childhood bullying victimization: evidence from a five-decade longitudinal British birth cohort. Am J Psychiatry 2014; 171: 777-84.

3 Van Dam NT, Rando K, Potenza MN, Tuit K, Sinha R. Childhood maltreatment, altered limbic neurobiology, and substance use relapse severity via trauma-specific reductions in limbic gray matter volume. JAMA Psychiatry 11 June 2014 (doi:10.1001/jamapsychiatry.2014.680).

4 Campanella $F$, Shallice $T$, Ius $T$, Fabbro $F$, Skrap M. Impact of brain tumour location on emotion and personality: a voxel-based lesion-symptom mapping study on mentalization processes. Brain 15 July 2014 (doi: 10.1093/brain/ awu183).

5 Kühn S, Gallinat J. Brain structure and functional connectivity associated with pornography consumption. The brain on porn. JAMA Psychiatry 2014; 71: 827-34.

6 Donoso M, Collins AGE, Koechlin E. Foundations of human reasoning in the prefrontal cortex. Science 2014; 344: 1481-6.

\title{
The temperance movement
}

\author{
Iain D. Smith
}

Imagine in today's culture of intoxication a people's movement arising to counter alcohol. The 19th century saw such a campaign. It had immense influence by the early 20th century. The temperance movement: an alliance of religious and political groups campaigning for personal and legislative change. The goal of moderation and the shunning of 'ardent spirits' soon evolved into advocacy of teetotalism - a term derived from a stammering ex-inebriate's advocacy of t-t-total abstinence. Individuals could 'Take the Pledge'. While Britain by the 1920s did not have prohibition, local areas could veto the sale of alcohol. The movement waned rapidly after WW2. 